\title{
Legal Status of the Strategic Environmental Assessment (SEA), Practice of Application and Challenges in Pakistan
}

Farasat Ali', Imran Ali Khan ${ }^{2}$, Waleed Asghar ${ }^{2 *}$, Zhenmei Liao ${ }^{2}$, Sobia Beghum ${ }^{3}$, Wim Douven ${ }^{4}$ and Kamran Ali Khan ${ }^{5}$

${ }^{1}$ Key State Laboratories, Nanjing Institute of limnology and Geography, University of Chinese Academy of Sciences, Nanjing, China

${ }^{2}$ School of Environment, Beijing Normal University, Beijing, China

${ }^{3}$ Karakoram International University, GilGit Baltistan, Pakistan

${ }^{4}$ UNESCO-IHE, Netherlands

${ }^{5}$ Comsats Institute of Information and Technology, Abbtobbad, Pakistan

\section{Retraction Note:}

The article entitled "Legal Status of the Strategic Environmental Assessment (SEA), Practice of Application and Challenges in Pakistan" has been accepted for publication in the International Journal of Waste Resources considering the statements provided in the article as personal opinion of the author which was found not having any conflict or biasness towards anything. As the article was a perspective one, information provided by the author was considered as an opinion to be expressed through publication.

Publisher took decision to make the article online solely based on the reviewers suggestion which considered the article not but a personal opinion of the author. However, it is found that the author have some personal concerns and issues, therefore, being retracted from the journal. 